\title{
Two-dimensional Yukawa interactions from nonlocal Proca quantum electrodynamics
}

\author{
Van Sérgio Alves, ${ }^{1}$ Tommaso Macrì, ${ }^{2,3,4}$ Gabriel C. Magalhães, ${ }^{1}$ E. C. Marino, ${ }^{5}$ and Leandro O. Nascimento ${ }^{3,6}$ \\ ${ }^{1}$ Faculdade de Física, Universidade Federal do Pará, \\ Av. Augusto Correa 01, Belém, PA, 66075-110, Brazil \\ ${ }^{2}$ Departamento de Física Teórica e Experimental, Universidade Federal do Rio Grande do Norte, \\ Natal-RN, Brazil \\ ${ }^{3}$ International Institute of Physics, Campus Universitário Lagoa Nova, \\ C.P. 1613, Natal RN, 59078-970, Brazil \\ ${ }^{4}$ Dipartimento di Scienze Fisiche e Chimiche, Università dell'Aquila, \\ via Vetoio, I-67010 Coppito-L'Aquila, Italy \\ ${ }^{5}$ Instituto de Física, Universidade Federal do Rio de Janeiro, \\ C.P. 68528, Rio de Janeiro, RJ, 21941-972, Brazil \\ ${ }^{6}$ Faculdade de Ciências Naturais, Universidade Federal do Pará, C.P. 68800-000, Breves, PA, Brazil
}

(Received 18 December 2017; published 3 May 2018)

\begin{abstract}
We derive two versions of an effective model to describe dynamical effects of the Yukawa interaction among Dirac electrons in the plane. Such short-range interaction is obtained by introducing a mass term for the intermediate particle, which may be either scalar or an abelian gauge field, both of them in $(3+1)$ dimensions. Thereafter, we consider that the fermionic matter field propagates only in $(2+1)$ dimensions, whereas the bosonic field is free to propagate out of the plane. Within these assumptions, we apply a mechanism for dimensional reduction, which yields an effective model in $(2+1)$ dimensions. In particular, for the gauge-field case, we use the Stueckelberg mechanism in order to preserve gauge invariance. We refer to this version as nonlocal-Proca quantum electrodynamics (NPQED). For both scalar and gauge cases, the effective models reproduce the usual Yukawa interaction in the static limit. By means of perturbation theory at one loop, we calculate the mass renormalization of the Dirac field. Our model is a generalization of Pseudo quantum electrodynamics (PQED), which is a gauge-field model that provides a Coulomb interaction for two-dimensional electrons. Possibilities of application to Fermi-Bose mixtures in mixed dimensions, using cold atoms, are briefly discussed.
\end{abstract}

DOI: 10.1103/PhysRevD.97.096003

\section{INTRODUCTION}

In the last decades, the interest of studying planar theories has increased in theoretical physics, mainly because of the discovery of new quantum effects, such as high- $T_{c}$ superconductivity, quantum Hall effect, and topological phase transitions [1]. Furthermore, the emergence of both massless and massive Dirac excitations in two-dimensional materials, such as graphene [2] and silicene [3], has built a bridge between high-energy and condensed matter physics. For instance, well-known effects have been experimentally verified, or proposed in reachable energy scales, see [4] for an experimental realization of

Published by the American Physical Society under the terms of the Creative Commons Attribution 4.0 International license. Further distribution of this work must maintain attribution to the author(s) and the published article's title, journal citation, and DOI. Funded by SCOAP.
Klein paradox in graphene. On the other hand, for quantum chromodynamics the interest relies on the possibility of studying confinement in simpler models [5]. More recently, ultracold atomic gases offered a clean and highly controllable platform for the quantum simulation of bosonic and fermionic systems [6]. Within such systems, static as well as dynamical properties of models in trapped geometries with short or long-range interactions [7] can be probed, for example via their collective dynamics, or via density or momentum correlations [8]. Importantly, prototypical high energy physics models can be mapped into the low-energy, nonrelativistic, many-body dynamics of ultracold atoms [9]. Recently the experimental demonstration of a digital quantum simulation of the paradigmatic Schwinger model, a $U(1)$-Wilson lattice gauge theory [10] was shown.

Among two-dimensional models, pseudo quantum electrodynamics (PQED) [11,12] (or reduced quantum electrodynamics [13]) has attracted some attention. This model describes the electromagnetic interaction in a system 
where electrons are confined to the plane, but photons (or the intermediating particle) may propagate out of the plane. Despite its apparent nonlocal nature, PQED is still unitary and local [14]. Indeed, its main striking feature is that the effective action, for the matter field, remains the very same as the one provided by quantum electrodynamics in $3+1$ dimensions, hence, unitarity is respected. In the static limit, it yields a Coulomb potential, which renormalizes the Fermi velocity in graphene [15], as it has been verified by experimental findings [16]. In the dynamical description, it is expected to generate a set of quantized-energy levels in graphene as well as an interaction driven quantum valley Hall effect [17] at low enough temperatures. Furthermore, chiral-symmetry breaking has been shown to take place for both zero and finite temperatures $[18,19]$. It also occurs in the presence of a Gross-Neveu interaction, whose main effect is to decrease the critical coupling constant, yielding a better scenario for dynamical mass generation [20]. Lower dimensional versions of PQED have been investigated in Ref. [21], aiming for applications in cold atoms and in Ref. [22] for applications in the realm of topological insulators. All of these works rely on the fact that PQED generates a long-range interaction in the static limit, namely, the Coulomb potential $V_{C}(r) \propto 1 / r$.

In the meson theory of Yukawa, the so-called Yukawa potential $V(r) \propto e^{-m r} / r$ is a static solution of the Green function equation $\left(-\nabla^{2}+m^{2}\right) V(r)=\delta(r)$, where $\delta(r)$ is the Dirac delta function [23]. Within the quantum-fieldtheory interpretation, we may claim that the mediating field has a mass term. Motivated by this well-known result, we shall use the paradigm of including a mass term, for the intermediate particle, in order to generate a short-range interaction, i.e., the Yukawa potential in the plane. This potential has been applied to describe bound states [24,25], electron-ion interactions in a crystal [26], and interactions between dark energy and dark matter [27] among others. Nevertheless, a planar quantum-field theory accounting for this interaction, for both static and dynamical regime, has not been derived yet.

In this paper, we show how one may include an interaction length in PQED, yielding a short-range and nonlocal theory. The simplest mechanism is to consider that the mediating particle, i.e., the boson field, is massive. This parameter has to be a consequence either of the coupling with a Higgs field in the broken phase or some sort of intrinsic parameter in the system. The latter case can indeed be realized experimentally in ultracold Bose-Fermi mixtures in mixed dimensions. The typical setup consists of a Bose-Einstein condensate trapped in a three-dimensional harmonic potential weakly interacting with a fermionic gas confined in a two-dimensional periodic potential (optical lattice). The bosonic field mass corresponds to the atomic mass of the atoms in a Bose-Fermi mixture. Here, we consider both the massive Klein-Gordon field as well as the massive Stueckelberg model. For both cases, we have a
Yukawa potential between static charges. This is exactly the kind of interaction that has been studied in Ref. [28-30] in the context of ultracold atomic gases. Thereafter, we calculate the mass renormalization at one loop in the smallcoupling limit.

The outline of this paper is the following: in Sec. II, we consider a Dirac field coupled to a scalar field, whose dynamics is given by the massive Klein Gordon model. In Sec. III, we introduce the gauge field model. Since a naive addition of a mass for the gauge field would break gauge invariance, we consider the well known Stueckelberg action. This model is a generalized version of Proca quantum electrodynamics, on which we perform the dimensional reduction. In Sec. IV, we compute the asymptotic behavior of the boson propagator at both small and large distances. In Sec. V, we show that, by tuning the mass of the intermediate field, one may control the sign of the quantum correction, generated by the electron-self energy. We also include one Appendix, where we present the details about the electron-self energy as well as the corrected gauge-field propagator at one loop in perturbation theory.

\section{THE SCALAR CASE}

In this section, we perform a dimensional reduction of the Yukawa action in $3+1$ dimensions. To generate an interaction length, we assume that the mediating particle is a massive real scalar field. Let us start with the Euclidean action in $(3+1)$ dimensions, given by

$$
\mathcal{L}_{4 \mathrm{D}}=\frac{1}{2} \partial_{\mu} \varphi \partial^{\mu} \varphi+\frac{1}{2} m^{2} \varphi^{2}+g \varphi \bar{\psi} \psi+\bar{\psi}\left(i \not \partial-M_{0}\right) \psi,
$$

where $g$ is a dimensionless coupling constant, $\varphi$ is a real and massive Klein-Gordon field, and $\psi$ is the Dirac field.

First, we calculate the effective action for the matter field $\mathcal{L}_{\text {eff }}[\psi]$. In order to do so, we define the vacuum functional $Z$

$$
\begin{aligned}
Z & =\int D \varphi D \bar{\psi} D \psi \exp \{-S[\psi, \varphi]\} \\
& =\int D \bar{\psi} D \psi D \varphi e \int d^{4} x\left[-\varphi\left(-\square+m^{2}\right) \varphi / 2-g \varphi \bar{\psi} \psi+O[\psi]\right] \\
& =\int D \bar{\psi} D \psi \exp \left\{-S_{\mathrm{eff}}\right\}
\end{aligned}
$$

Integrating out $\varphi$ in Eq. (2) yields

$\mathcal{L}_{\text {eff }}[\psi]=-\frac{g^{2}}{2} \int d^{4} x d^{4} y(\bar{\psi} \psi)(x) \Delta_{\varphi}(x-y)(\bar{\psi} \psi)(y)$,

where

$$
\left(-\square+m^{2}\right)^{-1} \equiv \Delta_{\varphi}(x-y)=\int \frac{d^{4} k}{(2 \pi)^{4}} \frac{e^{-i k(x-y)}}{k^{2}+m^{2}}
$$


is the free scalar-field propagator, which yields the interaction between the matter field. The static interaction $V(r)$ is provided by the Fourier transform of Eq. (4) at $k_{0}=0$ (no time dependence), namely,

$$
V(r)=\int \frac{d^{3} k}{(2 \pi)^{3}} \frac{e^{-i \mathbf{k} \cdot \mathbf{r}}}{\mathbf{k}^{2}+m^{2}}=\frac{e^{-m r}}{4 \pi r} .
$$

Equation (5) is the well-known Yukawa potential, where the inverse of $m$ is the interaction length of the model. This is just the consequence of the coupling $g \varphi \bar{\psi} \gamma \psi$ in $(3+1) \mathrm{D}$.

Next, we show how to apply the procedure of dimensional reduction of PQED to Eqs. (3) and (5). In other words, we consider the case where the fermionic field is confined to the plane, but the bosonic field is not. This is an approximation of the derivation of PQED [11]. In order to do so, we assume that matter field is confined to the plane, i.e.,

$$
\bar{\psi} \psi(x)=\bar{\psi} \psi\left(x_{0}, x_{1}, x_{2}\right) \delta\left(x_{3}\right) .
$$

Using Eq. (6) in Eq. (3), we obtain

$$
\mathcal{L}_{\text {eff }}=-\frac{g^{2}}{2} \int d^{3} x d^{3} y(\bar{\psi} \psi)(x) G_{\varphi}(x-y)(\bar{\psi} \psi)(y),
$$

where $G_{\varphi}(x-y)=\Delta_{\varphi}\left(x-y, x_{3}=0, y_{3}=0\right)$ is the effective scalar-field propagator in $(2+1)$ dimensions, given by

$G_{\varphi}(x-y)=\int \frac{d^{3} k}{(2 \pi)^{3}} e^{-i k(x-y)} \int \frac{d k_{z}}{(2 \pi)} \frac{1}{k^{2}+k_{z}^{2}+m^{2}}$.

Integrating over $k_{z}$ above, we find

$$
G_{\varphi}(x-y)=\int \frac{d^{3} k}{(2 \pi)^{3}} \frac{e^{-i k(x-y)}}{2 \sqrt{k^{2}+m^{2}}} .
$$

In the static limit, Eq. (9) yields the Yukawa potential. Indeed,

$$
V(r)=\int \frac{d^{2} k}{(2 \pi)^{2}} \frac{e^{-i \mathbf{k} \cdot \mathbf{r}}}{2 \sqrt{\mathbf{k}^{2}+m^{2}}}=\frac{e^{-m r}}{4 \pi r},
$$

as expected from our dimensional reduction.

We may go beyond the static approximation by considering a nonlocal model with a propagator equal to Eq. (9). This is given by

$\mathcal{L}_{3 D}=\frac{1}{2} \partial^{\mu} \varphi K[\square] \partial_{\mu} \varphi+\frac{1}{2} m^{2} \varphi^{2}+g \varphi \bar{\psi} \psi+\bar{\psi}\left(i \not \partial-M_{0}\right) \psi$,

with

$$
K[\square] \equiv \frac{2 \sqrt{-\square+m^{2}}}{-\square} \equiv \int \frac{d^{3} k}{(2 \pi)^{3}} e^{i k x} \frac{2 \sqrt{k^{2}+m^{2}}}{k^{2}} .
$$

From Eq. (11), it is straightforward that the scalar-field propagator is $G_{\varphi}$ and the effective action for the matter field are the very same as in Eqs. (9) and (7), respectively.

\section{THE GAUGE-FIELD CASE}

In this section, we consider that the matter field is coupled to a gauge field $A_{\mu}$, through a minimal coupling $A^{\mu} j_{\mu}$. The main steps are the same as in the previous calculation. Nevertheless, a massive term as $m^{2} A_{\mu} A^{\mu}$ breaks gauge invariance. Hence, we must be careful about how to introduce the mass, i.e., the length scale for interactions. For the sake of simplicity, we consider an Abelian field $A_{\mu}$, for which we may use the Stueckelberg mechanism. This is a mechanism for generating mass for $A_{\mu}$ without breaking gauge invariance [31]. Before we perform the dimensional reduction, let us summarize this method.

First, we introduce a mass term into $(3+1)$ QED, yielding the so-called Proca quantum electrodynamics, whose action is given by

$$
\begin{aligned}
\mathcal{L}_{4 \mathrm{D}}= & \frac{1}{4} F_{\mu \nu} F^{\mu \nu}+\frac{m^{2}}{2} A_{\mu} A^{\mu}+e A^{\mu} j_{\mu} \\
& -\frac{\lambda}{2}\left(\partial_{\mu} A^{\mu}\right)^{2}+\bar{\psi}\left(i \not \partial-M_{0}\right) \psi
\end{aligned}
$$

where $e$ is the electric charge, $j_{\mu}=\bar{\psi} \gamma_{\mu} \psi$ is the matter current, $m^{2}$ is a massive parameter for the gauge field, and $\lambda$ is a gauge-fixing parameter. As expected,

$$
\frac{m^{2}}{2} A_{\mu} A^{\mu} \rightarrow \frac{m^{2}}{2} A_{\mu} A^{\mu}+m A^{\mu} \partial_{\mu} B+\frac{1}{2}\left(\partial_{\mu} B\right)^{2}
$$

under $A_{\mu} \rightarrow A_{\mu}+\partial_{\mu} B / m$. Indeed, gauge invariance is explicitly broken.

Next, we introduce a scalar-field $B(x)$ (the Stueckelberg field) in Eq. (13), hence,

$$
\begin{aligned}
\mathcal{L}_{4 \mathrm{D}}= & \frac{1}{4} F_{\mu \nu} F^{\mu \nu}+\frac{m^{2}}{2}\left(A_{\mu}-\frac{\partial_{\mu} B}{m}\right)^{2}+e A^{\mu} j_{\mu} \\
& -\frac{\lambda}{2}\left(\partial_{\mu} A^{\mu}\right)^{2}+\bar{\psi}\left(i \not \partial-M_{0}\right) \psi .
\end{aligned}
$$

Equation (15) is known as Stueckelberg action. Despite the mass for the gauge field, it is invariant under gauge transformation, namely, $A_{\mu} \rightarrow A_{\mu}+\partial_{\mu} \Lambda, B \rightarrow B-m \Lambda$, and $\psi \rightarrow \exp (-i e \Lambda) \psi$ [31]. Furthermore, it still produces the Yukawa interaction between static charges. 
From Eq. (15), we may find

$$
\begin{aligned}
\mathcal{L}_{4 \mathrm{D}}= & \frac{1}{4} F_{\mu \nu} F^{\mu \nu}+\frac{m^{2}}{2} A_{\mu} A^{\mu}+\frac{\left(\partial_{\mu} B\right)^{2}}{2}+m A_{\mu} \partial^{\mu} B \\
& +e A^{\mu} j_{\mu}-\frac{\lambda}{2}\left(\partial_{\mu} A^{\mu}\right)^{2}+\bar{\psi}\left(i \not \supset-M_{0}\right) \psi .
\end{aligned}
$$

We shall use Eq. (16) to compute the effective action for the matter associated to the matter current $j_{\mu}$. The vacuum functional is

$$
Z=\int D \bar{\psi} D \psi D A_{\mu} D B \exp \left\{-S\left[\psi, A_{\mu}, B\right]\right\} .
$$

Integration over $B$ yields

$$
\begin{aligned}
\mathcal{L}_{4 \mathrm{D}}^{\mathrm{eff}}= & \frac{1}{4} F_{\mu \nu} F^{\mu \nu}+\frac{m^{2}}{2} A_{\mu} A^{\mu}+\frac{\lambda_{\square}}{2}\left(\partial_{\mu} A^{\mu}\right)^{2} \\
& +e A^{\mu} j_{\mu}+\bar{\psi}\left(i \not \partial-M_{0}\right) \psi,
\end{aligned}
$$

where

$$
\lambda_{\square}=-\lambda+m^{2} \square^{-1} .
$$

Now, for the sake of simplicity, we isolate the quadratic term in $A_{\mu}$, hence,

$$
\begin{aligned}
\mathcal{L}_{4 \mathrm{D}}^{\mathrm{eff}}= & \frac{1}{2} A^{\mu}\left[\left(-\square+m^{2}\right) \delta_{\mu \nu}+\lambda_{\square} \partial_{\mu} \partial_{\nu}\right] A^{\nu} \\
& +e A^{\mu} j_{\mu}+\bar{\psi}\left(i \not \partial-M_{0}\right) \psi .
\end{aligned}
$$

Integrating out $A_{\mu}$, we get our desired effective action

$$
\mathcal{L}_{4 \mathrm{D}}^{\mathrm{eff}}[j]=-\frac{e^{2}}{2} j^{\mu}(x) \Delta_{\mu \nu} j^{\nu}(y)+\bar{\psi}\left(i \not \partial-M_{0}\right) \psi,
$$

where

$$
\Delta_{\mu \nu}=\frac{1}{-\square+m^{2}}\left(\delta_{\mu \nu}-\frac{\lambda_{\square}}{-\square+m^{2}-\lambda_{\square}} \partial_{\mu} \partial_{\nu}\right) .
$$

Using Eq. (22) in Eq. (21) with charge conservation $\partial_{\mu} j^{\mu}=0$, we may conclude that, for a correct description, the gauge-field propagator is

$$
\Delta_{\mu \nu}(x-y)=\int \frac{d^{4} k}{(2 \pi)^{4}} e^{-i k(x-y)} \frac{\delta_{\mu \nu}}{k^{2}+m^{2}} .
$$

In this way, all the gauge-dependence vanishes in the effective action.

In order to obtain the projected theory in $(2+1)$ dimensions, we consider that the current matter only propagates in the plane, therefore,

$$
j^{\mu}(x)= \begin{cases}j^{\mu}\left(x_{0}, x_{1}, x_{2}\right) \delta\left(x_{3}\right), & \mu=0,1,2, \\ 0, & \mu=3 .\end{cases}
$$

Similarly to the previous case, this shall lead to

$$
G_{0, \mu \nu}=\int \frac{d^{3} k}{(2 \pi)^{3}} e^{-i k(x-y)} \frac{\delta_{\mu \nu}}{2 \sqrt{k^{2}+m^{2}}},
$$

which is the effective propagator in $(2+1)$ dimensions.

Our main goal is to find the corresponding theory in $(2+1)$ dimensions with the same effective action in Eq. (21). This model reads

$$
\begin{aligned}
\mathcal{L}_{3 \mathrm{D}}= & \frac{1}{2} F_{\mu \nu} K[\square] F^{\mu \nu}+\lambda A^{\mu} \partial_{\mu} K[\square] \partial_{\nu} A^{\nu}+e A^{\mu} j_{\mu} \\
& +\bar{\psi}\left(i \not \supset-M_{0}\right) \psi,
\end{aligned}
$$

where $\lambda$ is a gauge-fixing parameter. It is straightforward to show that, after integrating out $A_{\mu}$ in Eq. (26), we obtain the same effective action as in Eq. (21) with the constraint in Eq. (24). For an arbitrary $\lambda$, the free gauge-field propagator reads

$$
G_{0, \mu \nu}(k)=\frac{1}{2 \sqrt{k^{2}+m^{2}}}\left[\delta_{\mu \nu}+\frac{(1-\lambda)}{\lambda} \frac{k_{\mu} k_{\nu}}{k^{2}}\right] .
$$

There are two main features of Eq. (26): (a) The massive parameter $m$ is no longer a pole of the gauge-field propagator, hence, it can not be thought as a mass and (b) Gauge-invariance is explicitly respected, i.e., there is no need to deal with Stueckelberg fields. Indeed, we could set $B=0$ from the very beginning, which means starting with Proca quantum electrodynamics and, therefore, breaking of gauge invariance. Then, after dimensional reduction, the corresponding 3D theory is still the same as in Eq. (26) and that it is gauge invariant.

\section{ASYMPTOTIC BEHAVIOR OF BOTH SCALAR AND GAUGE-FIELD PROPAGATORS}

In this section, we calculate the asymptotic expressions of $G_{\varphi}(x-y ; m)$ and $\delta^{\mu \nu} G_{0 \mu \nu}(x-y ; m)$, i.e., propagators in the space-time coordinates. We use the integral version in Eq. (8), which has an extra $k_{z}$-integral. Hence,

$G_{\varphi}(x-y ; m)=\int \frac{d^{3} k}{(2 \pi)^{3}} e^{-i k(x-y)} \int_{-\infty}^{+\infty} \frac{d \mu}{(2 \pi)} \frac{1}{k^{2}+\mu^{2}+m^{2}}$.

Note that we have replaced $k_{z}$ by $\mu$, since $\mu$ is just a parametric variable. Thereafter, we use Eq. (5) for solving the $k$-sphere integral, therefore, 


$$
G_{\varphi}(x-y ; m)=\int_{-\infty}^{+\infty} \frac{d \mu}{(2 \pi)} \frac{1}{4 \pi|x-y|} e^{-\sqrt{\left(\mu^{2}+m^{2}\right)}|x-y|} .
$$

Next, after solving the $\mu$-integral (see Ref. [32]), we have

$$
G_{\varphi}(x-y ; m)=\frac{m}{4 \pi^{2}|x-y|} K_{1}(m|x-y|),
$$

where $K_{1}$ is a modified Bessel function of the second kind. In the short-range limit $m|x-y| \ll 1$, it yields

$$
G_{\varphi}(x-y ; m) \approx \frac{1}{4 \pi^{2}|x-y|^{2}},
$$

whereas in the long-range limit $m|x-y| \gg 1$, we find

$$
G_{\varphi}(x-y ; m) \approx \sqrt{\frac{m \pi}{2}} \frac{e^{-m|x-y|}}{4 \pi^{2}|x-y|^{3 / 2}} .
$$

The gauge-field propagator may be calculated by following the very same steps.

We have described some general features of NPQED, in particular, its derivation, two-point functions, and interactions. Next, we shall explore quantum corrections by using perturbation theory.

\section{PERTURBATION THEORY RESULTS}

In this section, we calculate the renormalized electron mass $M_{R}$ of the model in Eq. (26) at one-loop approximation. The details about the calculation are shown in Appendix A. In particular, we would like to obtain its dependence on $m$, the mass term of the gauge field. Note that in our 3D model, this parameter must to be understood as the inverse of the interaction length. This, nevertheless, is the mass of the intermediate field that propagates in 4D. Thereafter a standard calculation, we obtain

$$
Z_{R}=1+\frac{\alpha}{2 \pi} f(z)
$$

where $Z_{R} \equiv M_{R} / M_{0}, z \equiv m / M_{0}$, and

$f(z)=\int_{0}^{1} d x \frac{2+x}{\sqrt{1-x}} \ln \left[\sqrt{(1-x)\left(z^{2}-x\right)+x}\right]$.

From Eq. (34), it is clear that $f(z)=f(-z)$, therefore, the corrections are only dependent on the modulus of $m$. From now on, we assume $\alpha=1 / 137$. Next, we would like to address the effects of the $m$ parameter on $Z_{R}$. Surprisingly, for $|z| \leq z_{c} \approx 1.2$, the quantum correction $\alpha f(z) / 2 \pi$ is negative, while for $|z| \geq z_{c}$, they become positive and cross the free-energy level $M_{0}$, see Fig. 1 .

Let us calculate the energy gap of the renormalized state $\quad \delta_{R} E=E_{R}^{+}-E_{R}^{-}=M_{0}+M_{0} \alpha f_{x}(z) / 2 \pi-\left(-M_{0}-\right.$ $\left.M_{0} \alpha f(z) / 2 \pi\right)=2 M_{0}+M_{0} \alpha f(z) / \pi$, where $E_{R}^{+}>0$ and

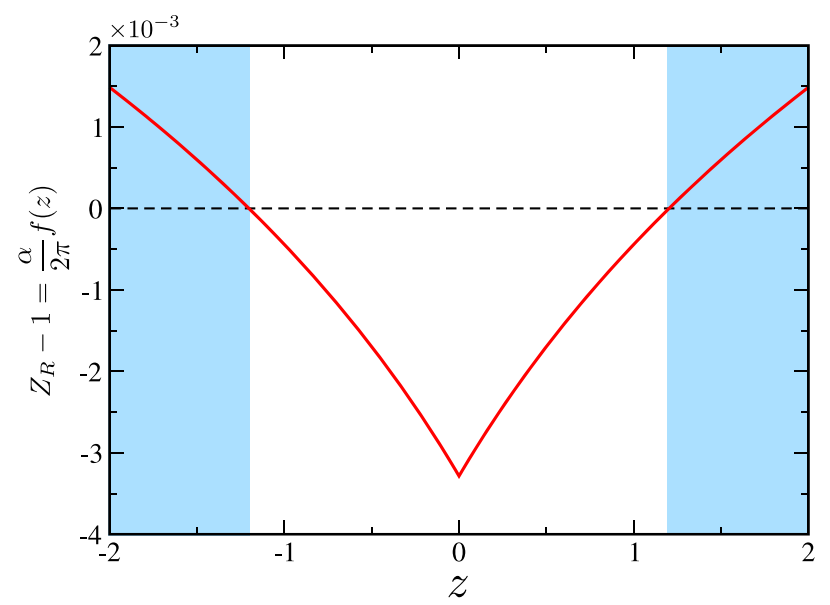

FIG. 1. Quantum correction for the electron mass. We plot the quantity $\alpha f(z) / 2 \pi$ in Eq. (33), where $z$ is the ratio between the inverse of interaction length $m$ and the bare electron mass $M_{0}$. For $|z| \leq z_{c} \approx 1.2$, we have $f(z)<0$, therefore, the renormalized energy gap $\delta_{R} E$ is less than the bare energy gap $2 M_{0}$. On the other hand, $|z| \geq z_{c}$ yields $f(z)>0$ (shaded area), i.e., the renormalized energy gap is larger than the bare energy gap. Finally, for $|z|=z_{c}$, we find $f\left(z_{c}\right)=0$ and the renormalized energy gap is equal to $2 M_{0}$.

$E_{R}^{-}<0$ are the positive and negative energies, see Appendix A. Since $f(z)$ may be negative for $|z| \leq z_{c} \approx 1.2$, we have that $\delta_{R} E$ lies inside the energy gap $2 M_{0}$. The case $z \leq z_{c}$ resembles the quantized energy levels calculated in Ref. [17] or Ref. [20], because that renormalized states are closer to the zero-energy level. Nevertheless, because we are in the small coupling limit, hence, we do not generate dynamical symmetry breaking, since $Z_{R} \rightarrow 0$ when $M_{0} \rightarrow 0$.

\section{DISCUSSION}

PQED has been applied to describe the interactions of two-dimensional electrons, in particular, graphene in the strong-coupling regime. The main results rely on the fact that electrons do interact trough the Coulomb potential, which has an infinite range. Here, nevertheless, we consider a scenario where interactions have a finite range. We have applied the very same procedure for deriving PQED [11], but considering a massive photon in $(3+1)$ dimensions. Our main result is that, after performing the dimensional reduction, one obtains that the matter field interacts through a Yukawa potential in the static limit. Similar to PQED, it yields a nonlocal theory in both space and time. Since the matter field is not relevant for the dimensional reduction, this model may be generalized to describe the Yukawa interaction between other kind of particles. Although our derivation follows standard steps of QED literature, we believe that they shall be relevant, in particular, for applications in condensed matter physics and cold-atom systems. 
In Ref. [28-30], the authors proposed a scheme applicable to a cold-atom mixture of bosons interacting with polarized fermions to study possible $p$-wave pairing in the fermionic component induced by the bosons. An essential ingredient of these proposals is the mixed dimensionality, namely the two-dimensional confinement of the fermionic species and the three-dimensional one for the bosons, reducing considerably trap losses from three-body effects. Interestingly, the static interaction between the fermionic particles results into a Yukawa potential allowing a notable increase in the critical temperature for the appearance of fermionic superfluidity. In this context, our study based on the NPQED may certainly be useful for the investigation of novel quantum phases starting from a fully relativistic fieldtheory approach that can be simulated in condensed matter platforms. Similarly, more recently it has been shown that a full dynamical description provides new results for PQED with applications to the exciton spectrum in transitionmetal dichalcogenides [33].

We finally discuss the importance of the order in which the following two operations are implemented, namely: (a) the inclusion of a mass for the gauge field; (b) the dimensional reduction. Interestingly, the result is sensitive to the order in which the inclusion of a mass and the dimensional reduction are performed. As a matter of fact, by doing "a" before "b," we have shown that the Yukawa potential $V(r)=e^{-m r} / 4 \pi r$ is obtained from the static limit of the gauge-field propagator, given by Eq. (27). This is proportional to $1 /\left(2 \sqrt{p^{2}+m^{2}}\right)$.

Conversely, starting with a massless gauge field, hence, by applying the dimensional reduction, we arrive at the PQED model [11], whose propagator is proportional to $1 /\left(2 \sqrt{p^{2}}\right)$. If we now couple the PQED model, to a Higgs field in the broken phase, such that a massive term is generated to the gauge field, this changes the propagator from $1 /\left(2 \sqrt{p^{2}}\right)$ to $1 /\left(2 \sqrt{p^{2}}+m\right)$, which clearly shows that we shall obtain a different model from the one associated to Eq. (26). The potential is now given by a combination of Coulomb and Keldysh [34] potentials, namely,

$$
V(r)=\frac{e^{2}}{4 \pi r}\left\{1-\frac{\pi r}{2 r_{0}}\left[\mathbf{H}_{0}\left(\frac{r}{r_{0}}\right)-Y_{0}\left(\frac{r}{r_{0}}\right)\right]\right\}
$$

where $r_{0} \equiv 2 / m, \mathbf{H}_{0}(m r)$, and $Y_{0}(m r)$ are Struve and Bessel functions, respectively. The potential in Eq. (35) does not decay exponentially at large distances as the Yukawa potential, rather, it has a power-law decay. We conclude, therefore, that we must be careful about how one wishes to use quantum electrodynamics in applications for lower-dimensional systems, because of this sensitive relation between the dimensional reduction and phenomenological parameters, such as a mass for the gauge field.

\section{ACKNOWLEDGMENTS}

We are grateful to Rodrigo Pereira for very interesting and stimulating discussions. G.C.M. acknowledges CAPES for financial support. T. M. acknowledges CNPq for support through Bolsa de produtividade em Pesquisa n.311079/2015-6. L.O.N. acknowledges the financial support from MEC/UFRN. E. C. M. is partially supported by $\mathrm{CNPq}$ and FAPERJ.

\section{APPENDIX: PERTURBATIVE RESULTS FOR GAUGE THEORY}

\section{Electron-self energy}

In this Appendix, we show some details about the electron-self energy. First, let us write the Feynman rules of Eq. (26) in Euclidean space. The free electronpropagator reads

$$
S_{0 F}=\frac{1}{\not p-M_{0}}=\frac{-\left(\not p+M_{0}\right)}{p^{2}+M_{0}^{2}},
$$

the gauge-field propagator (in the Feynman gauge $\lambda=1$ ) is

$$
G_{0, \mu \nu}(p)=\frac{\delta_{\mu \nu}}{2 \sqrt{p^{2}+m^{2}}},
$$

and the vertex interaction is $\Gamma^{\mu}=\gamma^{\mu} e$. The Euclidean matrices satisfy $\left\{\gamma_{\mu}, \gamma_{\nu}\right\}=-2 \delta_{\mu \nu}$. From Eq. (A1), we have that the pole of the free Dirac electron is $p_{E}^{2}=-M_{0}^{2}$, where $p_{E}^{2}$ is the Euclidean momentum. For calculating the physical mass, one must return to the Minkowski space, using $p_{E}^{2} \rightarrow-p_{M}^{2}$, such that $p_{M}^{2}=M_{0}^{2}$ are the physical poles.

The corrected electron propagator $S_{F}$ is

$$
S_{F}=S_{0 F}+S_{0 F}(\Sigma) S_{0 F}+\cdots
$$

therefore,

$$
S_{F}^{-1}=S_{0 F}^{-1}-\Sigma(p) .
$$

The electron-self energy reads

$$
\Sigma(p)=e^{2} \int \frac{d^{3} k}{(2 \pi)^{3}} \Gamma^{\mu} S_{0 F}(k) \Gamma^{\nu} G_{0, \mu \nu}(p-k) .
$$

Equation (A5) has a linear divergence, therefore, we need to use a regularization scheme. We choose to use the usual dimensional regularization $\epsilon=3-D$, where $D$ is an arbitrary dimension, which we shall consider $D \rightarrow 3$ in the very end of the calculation. After application of standard methods, we find 


$$
\Sigma(p)=2 C I+2 C\left(\ln \frac{\mu}{M_{0}}+\frac{1}{\epsilon}\right)\left(\frac{2 \not p}{3}-6 M_{0}\right),
$$

where $\mu$ is an arbitrary massive parameter, generated by the prescription $e \rightarrow e \mu^{\epsilon / 2}$, with $\epsilon=D-3$, where $D$ is the dimension of the space-time, as usually done in the dimensional regularization. The constant $C$ is given by

$$
C=\frac{e^{2}}{16 \pi^{2}}
$$

and the parametric integral reads

$$
I=\int_{0}^{1} d x \frac{\not p(x-1)+3 M_{0}}{\sqrt{1-x}} \ln \frac{\Delta}{M_{0}},
$$

with

$$
\Delta=\sqrt{p^{2} x(1-x)+M_{0}^{2} x+m^{2}(1-x)} .
$$

Equation (A6) has both finite and a regulator-dependent terms, which must be eliminated by some renormalization scheme. For simplicity, we choose the minimal subtraction procedure, which avoids the poles by introducing counterterms in the original action. Hence,

$$
\Sigma^{R}(p)=\lim _{\mu, \epsilon \rightarrow 0}(\Sigma(p, \mu, \epsilon)-C T)=A(p) \not p+B(p),
$$

where CT stands for counterterms,

$$
A(p)=2 C \int_{0}^{1} d x \frac{(x-1)}{\sqrt{1-x}} \ln \frac{\Delta}{M_{0}},
$$

and

$$
B(p)=2 C \int_{0}^{1} d x \frac{3 M_{0}}{\sqrt{1-x}} \ln \frac{\Delta}{M_{0}} .
$$

Using Eq. (A10) in Eq. (A4), we find

$$
S_{F}^{-1}(p)=\left[(1-A(p)) \not p-\left(M_{0}+B(p)\right)\right] .
$$

Multiplying Eq. (A13) by $(1+A(p))$, we have

$$
(1+A(p)) S_{F}^{-1}(p)=-i\left[\not p-\left(M_{0}+B(p)\right)(1+A(p))\right] .
$$

Next, we define the renormalized matter field $\psi_{R}$, namely,

$$
\psi_{R}=Z_{\psi}^{1 / 2} \psi=\sqrt{1-A(p)} \psi .
$$

Therefore, the physical propagator reads

$$
S_{R F}^{-1}=\frac{1}{\not p-M(p)}=\frac{-[\not p+M(p)]}{p^{2}+M^{2}(p)},
$$

with

$$
M(p)=M_{0}+B(p)+M_{0} A(p) .
$$

Using Eqs. (A7), (A12), and (A11) in Eq. (A17), we find

$$
M(p)=M_{0}+\frac{\alpha}{2 \pi} M_{0} \int_{0}^{1} d x \frac{(2+x)}{\sqrt{1-x}} \ln \frac{\Delta}{M_{0}} .
$$

Equation (A17) yields the so-called mass function $M(p)$, which is, essentially, the momentum-dependent part of the electron-self energy that renormalizes the electron mass [35]. In Fig. 2, we show that by using different values of $m / M_{0}$, we may generate quantum corrections that either increase or decrease the renormalized mass in comparison with the bare value $M_{0}$. To clarify this result, we shall calculate the renormalized mass $M_{R}$.

The pole of Eq. (A16) is given by the solution of $p_{E}^{2}=-M^{2}\left(p_{E}^{2}\right)$, which, in the Minkowski space, yields $m_{R}^{2}=M^{2}\left(-M_{R}^{2}\right)$, where $M_{R}^{2}$ is the renormalized mass by applying the substitution $p_{E}^{2} \rightarrow-M_{R}^{2}$ for calculating the pole. Furthermore, since we are at one-loop approximation, we may use $M\left(-M_{R}^{2}\right)=M\left(-M_{0}^{2}\right)$. Therefore, the renormalized masses are $M_{R}= \pm M\left(-M_{R}\right)=E_{R}^{ \pm}$, where $E_{R}^{+}=$ $+\left|M\left(-M_{R}\right)\right|$ and $E_{R}^{-}=-\left|M\left(-M_{R}\right)\right|$ are positive and negative solutions, respectively. Using Eq. (A17) with $M_{R}^{2}=M\left(-M_{0}^{2}\right)$ and $e^{2}=4 \pi \alpha$, we find

$$
\frac{\left|M_{R}\right|}{M_{0}}=1+\frac{\alpha}{2 \pi} \int_{0}^{1} d x \frac{2+x}{\sqrt{1-x}} \ln \left[\sqrt{(1-x)\left(\frac{m^{2}}{M_{0}^{2}}-x\right)+x}\right] .
$$

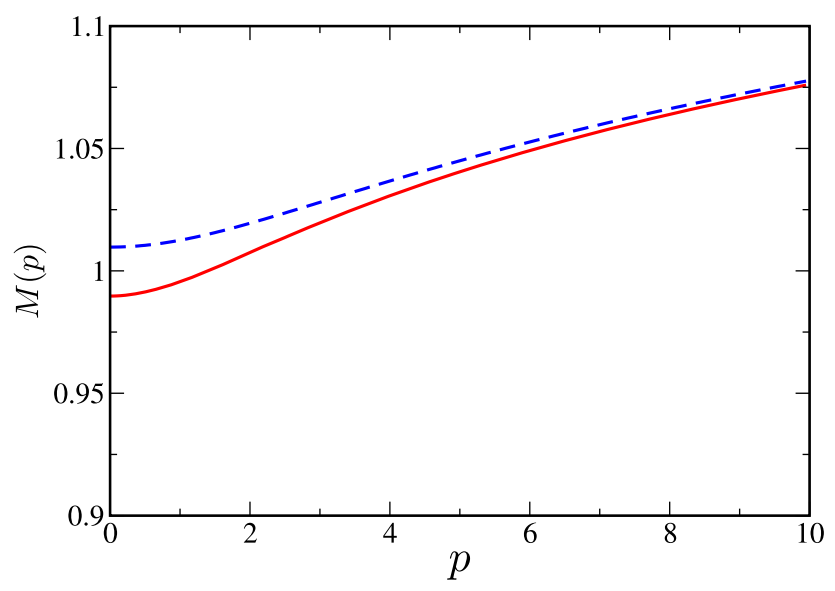

FIG. 2. Mass function $M(p)$ in Eq. (A17). For this plot we set $M_{0}=1$ and $\alpha=1 / 137$. Dashed line: $m / M_{0}=1.5$, full line: $m / M_{0}=0.5$. Note that $M(p=0)>M_{0}$ for the dashed line, but $M(p=0)<M_{0}$ for the full line. 
Considering $Z_{R} \equiv M_{R} / M_{0}$ and $z \equiv m / M_{0}$, we obtain Eq. (33) and Eq. (34). In the scalar-field case, given by Eq. (11), the critical point $z_{c}$ is the same. This is not surprising because of the similarities of the electron-self energy and the bosonic propagators.

Equation (A19) shows that the renormalized electron mass is dependent on $m$. It is reasonable to calculate whether $m$ itself is renormalized in one loop. For consistency, the renormalization of the boson mass $m$ in the Stueckelberg theory, in Eq. (16), would imply a renormalized mass parameter in the reduced model, given by Eq. (26). Within the Landau gauge $\lambda=\infty$, the corrected gauge-field propagator with the insertion of polarization tensor reads

$$
\begin{aligned}
G_{\mu \nu}(p) & =\frac{\delta_{\mu \nu}}{p^{2}+m^{2}+e^{2} \Pi(p)}, \\
& =\frac{\delta_{\mu \nu}}{p^{2}+m_{R}^{2}}
\end{aligned}
$$

where $m_{R}$ is the renormalized mass given by

$$
m_{R}^{2}=m^{2}+e^{2} \Pi\left(p=m_{R}\right),
$$

where $\Pi(p)$ is given by [36]

$\Pi(p)=\frac{p^{2}}{2 \pi^{2}} \int_{0}^{1} d x x(1-x)\left[\frac{2}{\epsilon}-\ln \frac{Q^{2}}{4 \pi \mu^{2}}-\gamma-\frac{1}{2}\right]$,

with $Q^{2}=M_{0}^{2}+x(1-x) p^{2}$. Thus, the bare mass of the gauge field is renormalized in one-loop approximation.
[1] B. A. Bernevig and T. Hughes, Topological Insulators and Topological Superconductors (Princeton University Press, Princeton, NJ, 2013); S.-Q. Shen, Topological Insulators Dirac Equation in Condensed Matter, Spring Series in SolidState Sciences (Springer, New York, 2012); M. Z. Hasan and C. L. Kane, Colloquium: Topological insulators, Rev. Mod. Phys. 82, 3045 (2010).

[2] K. S. Novoselov, A. K. Geim, S. V. Morozov, D. Jiang, Y. Zhang, S. V. Dubong, I. V. Grigorieva, and A. A. Firsov, Electric field effect in atomically thin carbon films, Science 306, 666 (2004).

[3] B. Lalmi, H. Oughaddou, H. Enriquez, A. Kara, S. Vizzini, B. Ealet, and B. Aufray, Epitaxial growth of a silicene sheet, Phys. Lett. 97, 223109 (2010).

[4] M. I. Katsnelson, K. S. Novoselov, and A. K. Geim, Chiral tunnelling and the Klein paradox in graphene, Nat. Phys. 2, 620(2006).

[5] T. Appelquist, M. J. Bowick, E. Cohler, and L. C. R. Wijewardhana, Chiral-Symmetry Breaking in $2+1$ Dimensions, Phys. Rev. Lett. 55, 1715 (1985); C. D. Roberts and A. G. Williams, Dyson-Schwinger equations and their application to hadronic physics, Prog. Part. Nucl. Phys. 33, 477 (1994); P. Maris, Influence of the full vertex and vacuum polarization on the fermion propagator in $(2+1)$ dimensional QED, Phys. Rev. D 54, 4049 (1996).

[6] I. Bloch, J. Dalibard, and W. Zwerger, Many-body physics with ultracold gases, Rev. Mod. Phys. 80, 885 (2008).

[7] F. Dalfovo, S. Giorgini, L. P. Pitaevskii, and S. Stringari, Theory of Bose-Einstein condensation in trapped gases, Rev. Mod. Phys. 71, 463 (1999); S. Giorgini, L. P. Pitaevskii, and S. Stringari, Theory of ultracold atomic Fermi gases, Rev. Mod. Phys. 80, 1215 (2008).For a detailed account of long-range interactions with ultracold atoms, polar molecules and dipolar gases: M. A. Baranov, M. Dalmonte, G. Pupillo, and P. Zoller, Condensed matter theory of dipolar quantum gases, Chem. Rev. 112, 5012 (2012); M. Saffman, T. G. Walker, and K. Mølmer, Quantum information with Rydberg atoms, Rev. Mod. Phys. 82, 2313 (2010); See also for more recent results on long-range hoppings as well as multi-body interactions: G. Gori, T. Macrì, and A. Trombettoni, Modulational instabilities in lattices with power-law hoppings and interactions, Phys. Rev. E 87, 032905 (2013); D. Laghi, T. Macrì, and A. Trombettoni, Excitations and stability of weakly interacting Bose gases with multibody interactions, Phys. Rev. A 96, 043605 (2017).

[8] L. Pitaevskii and S. Stringari, Bose-Einstein Condensation and Superfluidity (Oxford Science Publications, New York, 2016); G. de Rosi and S. Stringari, Collective oscillations of a trapped quantum gas in low dimensions, Phys. Rev. A 92, 053617 (2015); M. K. Tey, L. A. Sidorenkov, E. R. S. Guajardo, R. Grimm, M. J. H. Ku, M. W. Zwierlein, Y.-H. Hou, L. Pitaevskii, and S. Stringari, Collective Modes in a Unitary Fermi Gas across the Superfluid Phase Transition, Phys. Rev. Lett. 110, 055303 (2013); S. Chiacchiera, T. Macrì, and A. Trombettoni, Dipole oscillations in fermionic mixtures, Phys. Rev. A 81, 033624 (2010).

[9] E. Zohar, J. I. Cirac, and B. Reznik, Quantum simulations of lattice gauge theories using ultracold atoms in optical lattices, Rep. Prog. Phys. 79, 014401 (2016).

[10] E. A. Martinez et al., Real-time dynamics of lattice gauge theories with a few-qubit quantum computer, Nature (London) 534, 516 (2016).

[11] E. C. Marino, Quantum electrodynamics of particles on a plane, and the Chern-Simons theory, Nucl. Phys. B408, 551 (1993). 
[12] A. Kovner and B. Rosenstein, Kosterlitz-Thouless mechanism of two-dimensional superconductivity, Phys. Rev. B 42, 4748 (1990); N. Dorey and N. E. Mavromatos, $Q E D_{3}$ and two-dimensional superconductivity without parity violation, Nucl. Phys. B386, 614 (1992).

[13] S. Teber, Electromagnetic current correlations in reduced quantum electrodynamics, Phys. Rev. D 86, 025005 (2012); Two-loop fermion self-energy and propagator in reduced $Q E D_{3,2}$, Phys. Rev. D 89, 067702 (2014); A. V. Kotikov and $\mathrm{S}$. Teber, Two-loop fermion self-energy in reduced quantum electrodynamics and application to the ultrarelativistic limit of graphene, Phys. Rev. D 89, 065038 (2014); S. Teber and A. V. Kotikov, Interaction corrections to the minimal conductivity of graphene via dimensional regularization European Phys. Lett. 107, 57001 (2014).

[14] E. C. Marino, L. O. Nascimento, V. S. Alves, and C. M. Smith, Unitarity of theories containing fractional powers of the d'Alembertian operator, Phys. Rev. D 90, 105003 (2014).

[15] M. A. H. Vozmediano and F. Guinea, Effect of Coulomb interactions on the physical observables of graphene, Phys. Scr. T146, 014015 (2012); F. de Juan, A. G. Grushin, and M. A. H. Vozmediano, Renormalization of Coulomb interaction in graphene: Determining observable quantities, Phys. Rev. B 82, 125409 (2010).

[16] D. C. Elias, R. V. Gorbachev, A. S. Mayorov, S. V. Morozov, A. A. Zhukov, P. Blake, L. A. Ponomarenko, I. V. Grigorieva, K. S. Novoselov, F. Guinea, and A. K. Geim, Dirac cones reshaped by interaction effects in suspended graphene, Nat. Phys. 7, 701 (2011).

[17] E. C. Marino, L. O. Nascimento, V.S. Alves, and C. M. Smith, Interaction Induced Quantum Valley Hall Effect in Graphene, Phys. Rev. X 5, 011040 (2015).

[18] V. S. Alves, W. S. Elias, L. O. Nascimento, V. Juričić, and F. Peña, Chiral symmetry breaking in the pseudo-quantum electrodynamics, Phys. Rev. D 87, 125002 (2013).

[19] L. O. Nascimento, V. S. Alves, F. Peña, C. M. Smith, and E. C. Marino, Chiral-symmetry breaking in pseudoquantum electrodynamics at finite temperature, Phys. Rev. D 92, 025018 (2015).

[20] V.S. Alves, R. O. C. Junior, E. C. Marino, and L. O. Nascimento, Dynamical mass generation in pseudo quantum electrodynamics with four-fermion interactions, Phys. Rev. D 96, 034005 (2017).
[21] J. C. P. Barros, M. Dalmonte, and A. Trombettoni, Longrange interactions from $U(1)$ gauge fields via dimensional mismatch, arxiv:1708.06585.

[22] N. Menezes, G. Palumbo, and C. M. Smith, Conformal QED in two-dimensional topological insulators, Sci. Rep. 7, 14175 (2017); L. O. Nascimento, Introduction to topological phases and electronic interactions in $(2+1)$ dimensions, Braz. J. Phys. 47, 215 (2017).

[23] L. M. Brown, Hideki Yukawa, and the meson theory, Phys. Today 39, 55 (1986).

[24] J. P. Edwards, U. Gerber, C. Schubert, M. A. Trejo, and A. Weber, The Yukawa potential: Ground state energy and critical screening, arxiv:1706.0.9979.

[25] H. S. Bennett, Upper limits for the number of bound states associated with the Yukawa potential, J. Res. Natl. Bur. Stand. 86, 503 (1981).

[26] B. Y. Yavidov, S. S. Djumanov, and S. Dzhumanov, Mass of a lattice polaron from an extended Holstein model using the Yukawa potential, Phys. Lett. A 374, 2772 (2010).

[27] A. A. Costa, L. C. Olivari, and E. Abdalla, Quintessence with Yukawa interaction, Phys. Rev. D 92, 103501 (2015).

[28] M. A. Caracanhas, F. Schreck, and C. M. Smith, Fermi-Bose mixture in mixed dimensions, arxiv:1701.04702v3.

[29] Z. Wu and G. M. Bruun, Topological Superfluid in a FermiBose Mixture with a High Critical Temperature, Phys. Rev. Lett. 117, 245302 (2016).

[30] J. M. Midtgaard, Z. Wu, and G. M. Bruun, Topological superfluidity of lattice fermions inside a Bose-Einstein condensate, Phys. Rev. A 94, 063631 (2016).

[31] H. Ruegg and M. Ruiz-Altaba, The Stueckelberg field, Int. J. Mod. Phys. A 19, 3265 (2004).

[32] I. S. Gradstheyn and I. M. Ryzhik, Table of Integrals, Series and Products, 7th ed. (Academic Press, New York, 2007).

[33] E. C. Marino, L. O. Nascimento, V. S. Alves, N. Menezes, and C.M. Smith, Quantum-electrodynamical approach to the exciton spectrum in transition-metal dichalcogenides, arxiv: $1710.08960 \mathrm{v} 1$.

[34] L. V. Keldysh, Coulomb interaction in thin semiconductors, and semimetal films, JETP Lett. 29, 716 (1979).

[35] T. Appelquist, M. J. Bowick, E. Cohler, and L. C. R. Wijewardhana, Chiral-Symmetry Breaking $2+1$ Dimensions, Phys. Rev. Lett. 55, 1715 (1985).

[36] S. Weinberg, The Quantum Theory of Fields (Cambridge University Press, Cambridge, England, 1995). 